\title{
Psychoactive drugs and driving
}

\section{Vanita Parekh \\ Director, Clinical Forensic Medical Services, Canberra Hospital, ACT \\ Associate professor, Australian National University}

\section{Keywords}

driving under the influence, psychotropic drugs, traffic accidents

Aust Prescr 2019;42:182-5 https://doi.org/10.18773/ austprescr.2019.070

\section{SUMMARY}

Any drug or substance with effects on the central nervous system can impair the ability to drive safely.

When prescribing, consider the effects of each drug on driving as well as the use of other substances. Advise the patient of the risks.

Opioids, benzodiazepines, anticonvulsants, antipsychotics and sedating antidepressants increase the risk of crashing. Erratic use of sedatives causes a higher level of impairment than stable regimens.

Patients who have complex medical conditions and take multiple drugs should undergo a fitnessto-drive assessment.

\section{Introduction}

Any psychoactive substance acting on the central nervous system can impair driving skills. These substances may be prescribed drugs, alcohol or substances of misuse. Alcohol, cannabis, opioids, stimulants and sedating drugs, such as benzodiazepines, are the substances of greatest concern in road safety.

While alcohol use has been declining, there has been an increase in the detection of other impairing substances in drivers. Misuse of prescription drugs is a concern, especially in relation to deaths from opioid use. Drugs affecting the ability to drive safely that have an increasing profile in motor vehicle collisions include pregabalin and gabapentin. Gabapentinoids may be being misused for their euphoric and dissociative effects. ${ }^{1,2}$

In Victoria, prescription drugs were involved in approximately $21 \%$ of fatal road collisions from 2007-2013. The prevalence varies in other states and territories depending on jurisdictional practice and data collection. ${ }^{3}$

\section{The tasks of driving}

Driving is a complex task that places demands on vision, rapid decision making, planning, tracking, vigilance, reaction time, coordination and gross motor activity. Most importantly, driving requires the ability to divide attention between several competing demands on cognitive skill. Any of these functions can be adversely affected by psychoactive substances either alone or in combination.

Alcohol is the most thoroughly understood drug with regard to driving ability. It is often used as a benchmark for drug effects, even though it has very little similarity with the pharmacokinetics and dynamics of other drugs.

\section{Random testing}

At present, random roadside alcohol and drug testing occurs to a variable extent throughout Australia. Alcohol testing involves a screening breath analysis followed by confirmatory testing with highly sensitive and specific instruments. When a confirmatory sample cannot be obtained, blood testing may be required.

Random roadside samples of saliva are tested for three substances:

- delta-9-tetrahydrocannabinol (cannabis)

- methamphetamine (commonly known as ice or speed)

- 3,4-methylenedioxymethylamphetamine (MDMA, commonly known as ecstasy).

These drugs were originally selected to avoid legal defences based on prescribed medicines. Screening is done at the roadside with an oral wipe. If the result is positive, an oral fluid sample is taken and sent for confirmatory testing. Cut-off concentrations for driving offences are set according to the Australian Standard (AS/NZS 4760-2019) or local policies.

\section{Mandatory testing}

Mandatory testing is required following a motor vehicle collision. Blood, and in some jurisdictions, urine, is collected. Samples are screened for commonly used substances including alcohol. If there is a positive indication, confirmatory tests are carried out. Observation of impaired driving behaviour in conjunction with blood sampling is generally required to prove the offence of driving under the influence, particularly for prescription drugs. Drug concentrations on their own do not predict individual driving ability due to the user developing tolerance to the drug. 


\section{Medical conditions}

The underlying medical condition for which a drug is prescribed may also have potential effects on driving ability. It is common in drivers with chronic conditions for there to be multiple effects on driving ability due to the illnesses and the drugs used to treat them. ${ }^{4}$ For further information on medical conditions and driving, refer to Austroads: Assessing Fitness to Drive. ${ }^{5}$ Many of the drug information databases used by doctors and pharmacists provide advice on the effects of drugs on driving. The more stringent requirements for commercial drivers are given in the Austroad standards.

\section{Depression}

Depression impairs driving skills such as cognitive abilities and concentration. It slows reaction times and can therefore increase the risk of a crash. ${ }^{6}$ Sedating antidepressant drugs, especially the tricyclic antidepressants, are likely to contribute to the increased risk. This risk decreases after six weeks, but not necessarily to the normal baseline level of control.?

\section{Epilepsy}

Most studies report an increased crash risk in people with epilepsy. ${ }^{8}$ Anticonvulsants frequently have adverse effects, such as fatigue, incoordination and dizziness, with the potential to impair driving ability. Starting or changing treatment including withdrawal of anticonvulsants alters the risk of seizures. Discussion and recommendation with regard to not driving and driving-free periods should be provided to the patients preferably in a written form with clear documentation in the prescriber's and dispenser's records. Providing a copy of the Austroad standards relevant to epilepsy is a useful adjunct to management.

\section{Alcohol}

Alcohol is the most common substance involved in road traffic collisions and deaths. Like most sedating drugs, alcohol impairs the ability to drive by increasing reaction time and decreasing concentration, coordination and tracking. It also increases risk-taking behaviour as drivers overestimate their skills. Alcohol is the only substance for which there is a generally accepted relationship between blood concentrations and the risk of crashing.

Alcohol interlocks are breath analysis devices installed in a vehicle. They require the driver to provide a breath sample before driving and at random times during a drive. Interlocks are useful for ensuring chronic alcohol users do not drive while intoxicated but they are expensive. In most jurisdictions their use cannot be made a condition for holding a driving licence unless there is a court order.

\section{Sedatives}

For sedating drugs, particularly benzodiazepines and opioids, the risk of having a motor vehicle collision is increased in the first four weeks after starting treatment and especially when combined with alcohol. Anticonvulsants, antidepressants and antipsychotics can also have sedative effects with the potential to affect driving ability. The prescriber and pharmacist must warn patients of these effects. ${ }^{9}$

An increased dose of any sedating substance will increase crash risk as will the absence of tolerance. There are other factors that affect the ability to drive safely such as the initiation period, time to steady state and effect, pre-existing medical conditions, driver experience and combinations with other substances.

Stabilisation on sedative drugs will generally take 6-8 weeks. In certain circumstances tolerance may develop, decreasing the crash risk from sedating drugs taken on a regular dosing schedule as long as other substances or alcohol are not used. The intermittent and erratic use of sedating substances can lead to unsafe driving.

Patients on a stable maintenance dose of opioid replacement therapy, such as methadone, will develop a tolerance to its sedating effects. They are usually safe to drive providing the opioid replacement therapy is taken as directed and no psychoactive drugs are taken with it.

\section{Stimulants}

Epidemiological data show increased rates of crashes, injuries and fatalities when methamphetamine ${ }^{10}$ or cocaine is present. The stimulant effects may be manifested by speeding, running red lights, aggressive driving, and unsafe overtaking and lane changes. There is no substantial evidence of sustained improvement in performance with methamphetamine or cocaine from epidemiological or on-road driving studies.

The effects of stimulants are biphasic. After the initial stimulatory phase, a period of extreme fatigue may ensue. There is depression and irresistible sleepiness which can reduce cognitive ability and cause drivers to fall asleep suddenly.

While a single low-dose stimulant may increase mental and motor performance in those who are sleep deprived or fatigued, it does not enhance performance in other people. It may improve performance in simple tasks, but not complex divided attention tasks such as driving. As with many other drugs there is no consistent relationship between the blood concentration of a stimulant and the degree of stimulation or crash risk. 
Amphetamines are excreted into saliva from blood. They may therefore be present in oral fluid at higher concentrations than in blood.

\section{Medicinal cannabis}

Medicinal cannabis was initially intended to only contain cannabidiol which is not psychoactive. Most patients with the conditions for which medical cannabis was originally claimed to be effective are not likely to be driving. Despite this, some medicinal cannabis does contain tetrahydrocannabinol (THC), especially if it has not been obtained from approved sources. THC is known to adversely affect driving skills but, because of its particular pharmacokinetics, its concentration in body fluids is not reliably related to impairment. Its presence at any level is associated with an increased crash risk." The laws relating to THC and driving are therefore not, and have never been, intended to be based on impairment but only on use of an illicit substance. It is illegal to drive with $\mathrm{THC}$ in the body regardless of prescription. The person is likely to be impaired in their ability to drive safely and:

- may not be insured in the event of a collision

- may be charged with criminal offences in the event of a collision

- may be charged with having a proscribed substance in their blood while driving.

THC is unlikely to be secreted into saliva from blood to any extent. Oral fluid tests can be positive because of residual THC deposited from cannabis smoke passing through the mouth, but they should not remain positive for more than a few hours after smoking.

The Therapeutic Goods Administration advises that 'Patients should not drive or operate machinery while being treated with medicinal cannabis'.

\section{Combination of substances}

It is common to find more than one drug in the body of surviving or dead drivers after a crash. Australian self-reports suggest that the prevalence of drugged driving in conjunction with alcohol may be as high as $4.1 \%$ with cannabis, $2.2 \%$ with ecstasy, $1.9 \%$ with methamphetamine and $0.9 \%$ with benzodiazepines. ${ }^{12}$ Co-administration of sedative drugs may cause more profound sedation and effects on driving ability than any drug taken on its own. The synergistic effects of alcohol and cannabis produce greater impairments in driving ability than each substance individually. ${ }^{13,14}$ This is due to the effects on different aspects of executive functioning such as cognition, and psychomotor and actual driving performance.
Prescribing regimens that have the least impairing effects on driving ability should always be considered. If prescribing multiple sedating drugs, for example the addition of metoclopramide for acute nausea or sedating antihistamines for hay fever, to other sedative substances, patients must be warned about the effects on driving ability. Cessation of driving may need to be recommended if it is not possible to change to a non-sedating drug.

\section{After effects of drug use}

Many substances impair safe driving ability, not only at the time of use, but also afterwards because of a hangover effect. Patients should be warned of these after effects particularly with alcohol and the benzodiazepines, opioids and stimulant drugs.

\section{Rehabilitation}

Patients can return to driving after drug or alcohol rehabilitation. At present the Austroads standard requirements ${ }^{5}$ are for a conditional licence after a month of remission and an absence of cognitive impairments relevant to driving. There should also be no end-organ effects that impact on driving.

\section{Reporting requirements}

Document all discussions with patients with regard to advice on driving and restrictions. Reporting requirements are detailed in Appendix 3 of the Austroads guidelines. Public safety issues must be considered for drivers who are unfit to drive due to medical or medicinal reasons, particularly those patients who may not self-report. In general, no penalties are applied to a prescriber who reports a patient, providing there is no malicious intent.

\section{Conclusion}

Driving requires a high level of skill. This may be adversely affected by medical conditions and the drugs used in treatment. Alcohol and illicit substances also impair the ability to drive safely.

When prescribing, consider the effects of the treatment on driving ability. Also enquire about the use of alcohol and other substances. Advise the patient when driving should be avoided or if extra precautions are required. $<$

Vanita Parekh is Director of the ACT Fitness to Drive Medical Clinic 


\section{REFERENCES}

1. Schjerning $O$, Rosenzweig $M$, Pottegård A, Damkier $P$, Nielsen J. Abuse potential of pregabalin: a systematic review. CNS Drugs 2016;30:9-25. https://doi.org/10.1007/ s40263-015-0303-6

2. Molero Y, Larsson H, D’Onofrio BM, Sharp DJ, Fazel S. Associations between gabapentinoids and suicidal behaviour, unintentional overdoses, injuries, road traffic incidents, and violent crime: population based cohort study in Sweden. BMJ 2019;365:I2147. https://doi.org/10.1136/bmj.I2147

3. Drummer $\mathrm{OH}$, Yap S. The involvement of prescribed drugs in road trauma. Forensic Sci Int 2016;265:17-21. https://doi.org/ 10.1016/j.forsciint.2015.12.050

4. Lacherez P, Wood JM, Anstey KJ, Lord SR. Sensorimotor and postural control factors associated with driving safety in a community-dwelling older driver population. J Gerontol A Biol Sci Med Sci 2014;69A:240-4. https://doi.org/10.1093/gerona/glt173

5. Austroads. Assessing fitness to drive: for commercial and private vehicle drivers, 2016. Austroads: Sydney; 2017. https://austroads.com.au/drivers-and-vehicles/assessingfitness-to-drive

6. Wickens CM, Smart RG, Mann RE. The impact of depression on driver performance. Int J Ment Health Addict 2014;12:524-37. https://doi.org/10.1007/s11469-014-9487-0

7. Dassanayake T, Michie P, Carter G, Jones A. Effects of benzodiazepines, antidepressants and opioids on driving: a systematic review and meta-analysis of epidemiological and experimental evidence. Drug Saf 2011:34:125-56. https://doi.org/10.2165/11539050-000000000-00000

8. Charlton J, Koppel S, Odell M, Devlin A, Langford J, O'Hare M, et al. Influence of chronic illness on crash involvement of motor vehicle drivers: 2 nd edition. Clayton (Vic.): Monash University Accident Research Centre; 2010. http://monashuniversity. mobi/muarc/reports/muarc300.html [cited 2019 Nov 1]
9. Wolff K, Brimblecombe R, Forfar JC, Forrest AR, Gilvarry E, Johnston A, et al.; Expert Panel on Drug Driving. Driving under the influence of drugs. London: UK Department for Transport; 2013. https://assets.publishing.service.gov.uk/ government/uploads/system/uploads/attachment data/ file/167971/drug-driving-expert-panel-report.pdf [cited 2019 Nov 1]

10. Logan BK. Methamphetamine and driving impairment J Forensic Sci 1996;41:457-64. https://doi.org/10.1520/ JFS13935J

11. Drummer $\mathrm{OH}$, Gerostamoulos J, Batziris H, Chu M, Caplehorn J, Robertson MD, et al. The involvement of drugs in drivers of motor vehicles killed in Australian road traffic crashes. Accid Anal Prev 2004;36:239-48. https://doi.org/ 10.1016/S0001-4575(02)00153-7

12. Mallik J, Johnston J, Goren N, Kennedy V. Drugs and driving in Australia. A survey of community attitudes, experience and understanding. Melbourne: Australian Drug Foundation; 2007.

13. Sewell RA, Poling J, Sofuoglu M. The effect of cannabis compared with alcohol on driving. Am J Addict 2009;18:185-93. https://doi.org/10.1080/10550490902786934

14. Downey LA, King R, Papafotiou K, Swann P, Ogden E, Boorman M, et al. The effects of cannabis and alcohol on simulated driving: Influences of dose and experience. Accid Anal Prev 2013;50:879-86. https://doi.org/10.1016/ j.aap.2012.07.016 